\title{
Star Formation in the Milky Way: The TRIFID Nebula (M20) from Herschel to Near-Infrared
}

\section{Paolo Persi ${ }^{* \dagger}$}

INAF/IAPS, Roma, Italy

E-mail: paolo.persieiaps.inaf.it

\section{Franco Giovannelli}

INAF/IAPS, Roma, Italy

\section{Davide Elia}

INAF/IAPS, Roma, Italy

\section{Lola Sabau-Graziati}

INTA, Madrid, Spain

\section{Carlos Roman-Zuniga}

UNAM, Instituto de Astronomia, Ensenada, Mexico

\section{MauricioTapia}

UNAM, Instituto de Astronomia, Ensenada, Mexico

\begin{abstract}
A preliminary study of the complex star forming region Trifd Nebula (M20) including Herschel far infrared images and new near infrared observations in $J H K s$ broad-band filters and $\mathrm{H}_{2}$ and Kc narrow band filters is presented. In particular we analyzed in detail four dense condensations and we derive several physical parameters.
\end{abstract}

XI Multifrequency Behaviour of High Energy Cosmic Sources Workshop

25-30 May 2015

Palermo, Italy

*Speaker.

${ }^{\dagger}$ A footnote may follow. 


\section{Introduction}

The Trifid Nebula (M20) is a young HII region with an age of $310^{5} \mathrm{yr}$ ionized by the O7V star HD164492 located at a distance of $1.68 \mathrm{Kpc}$ in the Sagittarium spiral arm. The Infrared Space Observatory (ISO) and the Hubble Space Telescope (HST ) ([1], [2],[3]) show the Trifid to be a dynamic,pre-Orion star-forming region containing young stellar objects ( YSOs) undergoing episodes of violent mass ejection, as well as protostars ( like HH 399) losing mass and energy to the nebula in jets.

From the Spitzer IRAC and MIPS images of the Trifid Nebula (M20) about 160 young stellar objects at different evolutionary stages have been identified by [4], while maps at millimeter, and submillimeter wavelengths have detected thirty three cores (TC=Trifid Condensation) which cover all the stages of proto-stellar evolution, from the early pre-stellar to the late proto-planetary phases[5].

Finally, 304 X-ray sources, have been detected by Chandra satellite, 30\% of which are hard sources and $70 \%$ of which have near infrared counterparts. X-ray emission are detected from TC 1 and possibly TC 4 , two massive star-forming cores with bipolar wings and associated Class 0 candidates[6].

The state-of-the-art for the Trifid star-forming region is presented in a review paper [7].

In order to study in more detail this complex star- forming region, we present here far-IR images from the Herschel Infrared Galactic Plane Survey (HI-GAL; [8]), and new near-infrared images obtained in broad and narrow-band filters. The observations are described in Sect.2, while in Sect.3 we present the results relative to five of the detected dense cores. Finally Sect.4 lists our conclusions.

\section{Observations}

\subsection{Herschel Infrared Galactic Plane Survey images}

The Trifid Nebula has been observed in the far-IR as part of the Herschel Infrared Galactic Plane survey (HI-GAL[8]) at 70,160, 250, 350 and $500 \mu \mathrm{m}$. The images were reduced using the HI-GAL standard pipeline [9]. From the images, we performed the sources extraction and photometry using the Curvature Threshold Extractor package (CuTEx, [10]), independently at each band. Figure 1 illustrates the Herschel three-colours image of an area of $20^{\prime} \times 20^{\prime}$ around the Trifid Nebula, composed from the $70 \mu \mathrm{m}$ (blue), $250 \mu \mathrm{m}$ (green) and $500 \mu \mathrm{m}$ (red) single images.

At least 53 dense cores have been detected. The brightest have been identified with the cores found at millimeter wavelength [5] as illustrated in Fig.1. In addition filament structures are present in the nebula.

\subsection{Near-IR observations}

Near infrared images in the JHKs broad-band filters and in the $\mathrm{H}_{2}(\lambda=2.122 \mu \mathrm{m})$ and the nearby Kc narrow-band filter of the Trifid Nebula, have been obtained during the nights of June 21 and 232013 using the camera OMEGA2000 attached at the 3.5m telescope of Calar Alto observatory. The camera uses the $2 \mathrm{~K} \times 2 \mathrm{~K}$ HAWAII- 2 array with a pixel scale of $0.45 \mathrm{arcsec} / \mathrm{pix}$, and the 


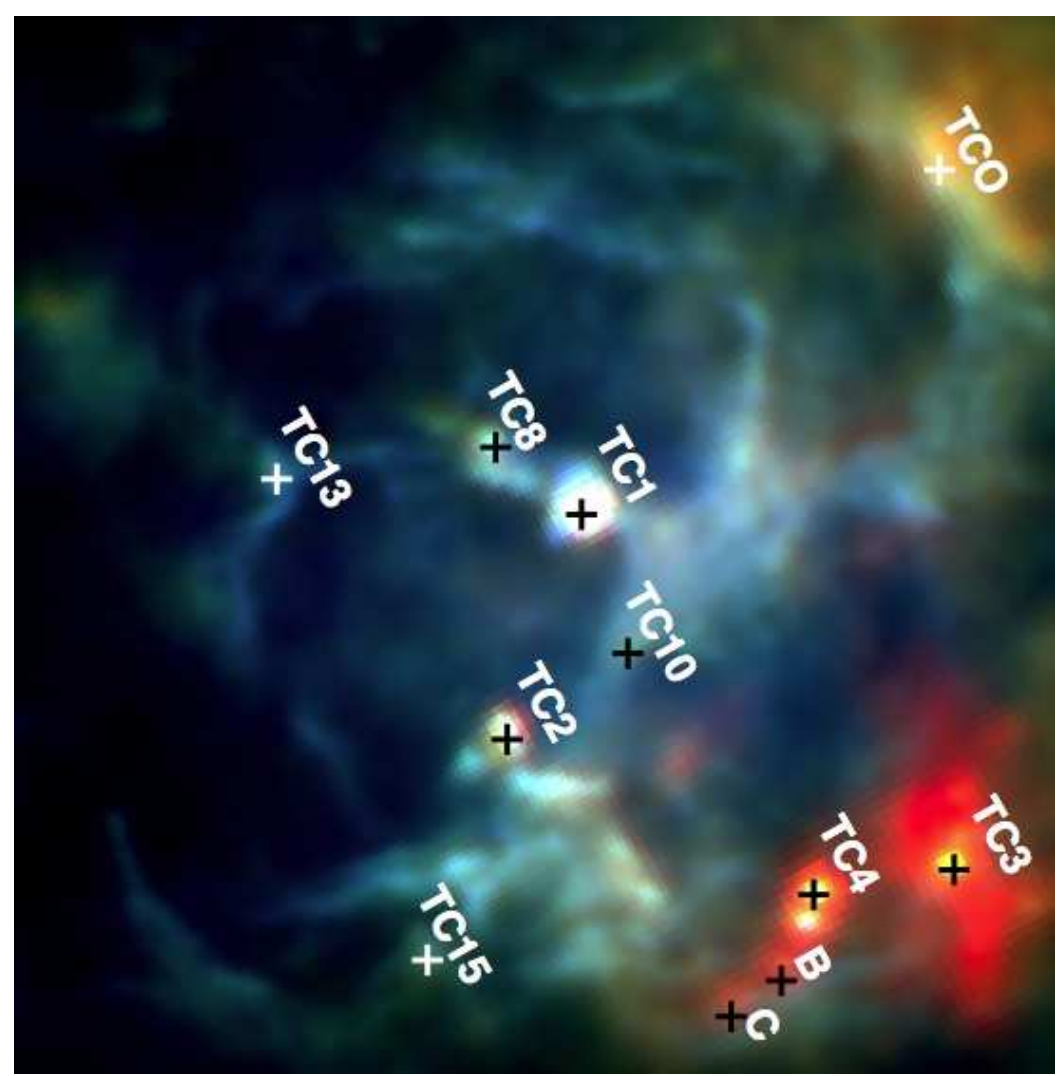

Figure 1: $20^{\prime} \times 20^{\prime}$ Herschel true-colour image made from $70 \mu \mathrm{m}$ (blue), $250 \mu \mathrm{m}$ (green), and $500 \mu \mathrm{m}$ (red) images of the Trifid Nebula. North is at the top and east to the left.

characteristics are given in [11].

The near-IR images relative to five dense cores will be discussed in the next section.

\section{Discussion}

We have derived the mass and temperature of the dense cores fitting the Herschel fluxes with a single- temperature modified blackbody of the type:

$$
F_{v}=\frac{M k_{0}}{d^{2}}\left(\frac{v}{v_{0}}\right)^{\beta} B_{v}(T),
$$

where $M, d$ and $T$ are the mass, the distance and the temperature of the envelope, $k_{0}=0.1$ $\mathrm{cm}^{2} \mathrm{~g}^{1}$ at $v_{0}=1200 \mathrm{GHz}$, already including a gas-to-dust ratio of 100 ; finally, the dust emissivity exponent $\beta$ has been kept constant $(\beta=2)$ to reduce the number of free parameters of the fit. Assuming a distance $\mathrm{d}=1.68 \mathrm{Kpc}$ for the Trifid Nebula, the derived masses and temperatures for five dense cores are reported in Table 1.

In the next subsections we will discuss individually the characteristics of these five dense cores. 
Table 1: Masses and temperatures of five dense cores of the Trifid Nebula

\begin{tabular}{lcc}
\hline Dense Core & $\begin{array}{c}\mathrm{M} \\
\end{array}$ & $\mathrm{T}_{d}$ \\
& Msun & $K$ \\
\hline $\mathrm{TC} 0$ & 90.87 & 13.0 \\
$\mathrm{TC} 1$ & 105.54 & 16.0 \\
$\mathrm{TC} 2$ & 37.35 & 18.8 \\
$\mathrm{TC} 3$ & 179.93 & 14.8 \\
$\mathrm{TC} 4$ & 104.15 & 17.8 \\
\hline
\end{tabular}
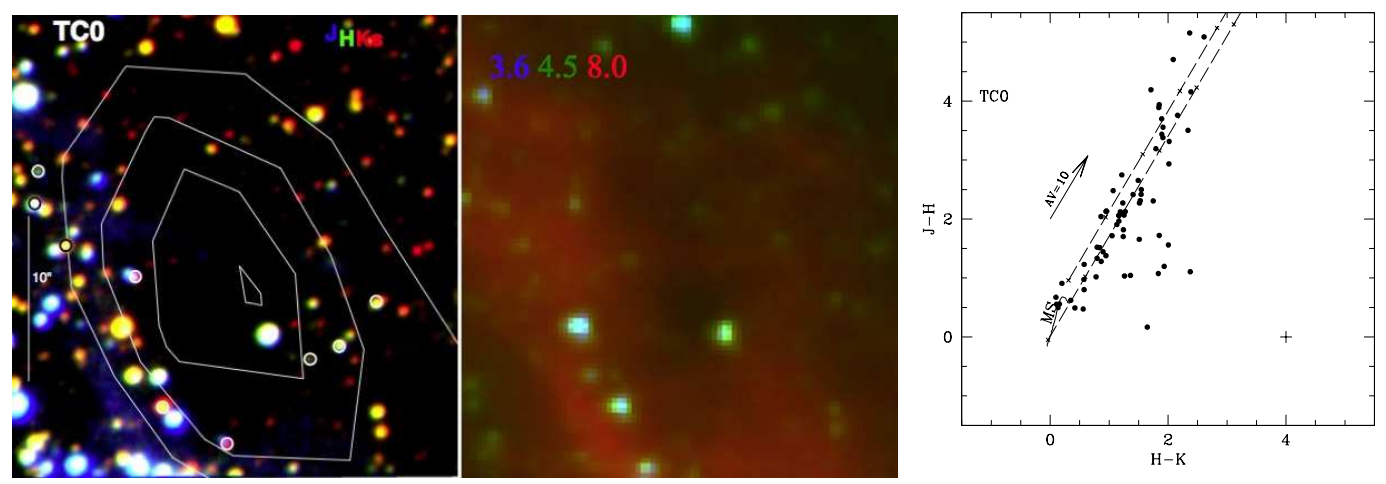

Figure 2: Left panel: $J H K_{S}$ (blue, green, red) and IRAC/Spitzer true color images of a region of size $1^{\prime} \times 1^{\prime}$ around TC0. North is at the top and east to the left. The contours are the $250 \mu \mathrm{m}$ Herschel emission. Right panel: $J-H$ versus $H-K_{s}$ diagram of the near-IR sources observed in TC0.

\subsection{The Trifid condensation TC0}

According to the millimeter observations [5] TC0 is the densest starless cores (several $10^{5}$ $\mathrm{cm}^{-3}$ ), currently hit by the ionization front, and may be the site for the next generation of stars. In Figure 2(Left panel) is reported our JHKs three-colour image of TC0 compared with the Spitzer/IRAC true color image. We have obtained the photometry in a field of $1^{\prime} \times 1^{\prime}$ around the central position of TC 0 . From our photometry we have derived the $J-H$ versus $H-K s$ diagram (Fig.2 (Right panel). Analyzing the diagram, we have found at least nine sources with near-IR excess . These young proto-stellar objects are located at the border of $\mathrm{TC} 0$, while no young objects are present inside the dense core. This is in agreement with the millimeter observations and define $\mathrm{TC} 0$ as a prestellar core.

\subsection{The Trifid condensation TC1}

Around the central position of the TC1 dense core, we have detected a young stellar cluster as illustrated in Figure 3. Three of these sources with near-IR excess here named TC1A,TC1B and TC1C have been detected also by Spitzer. TC1A is at the same position of the far-R and millimeter peak. Combining the observed flux densities from near-IR to millimeter we have derived the spectral energy distribution (SED) of this source illustrated in Figure 4(Left panel).

In order to derive the physical parameters of this source, the SED has been fitted with the infalling envelope+disc+central source radiation transfer model [12] by using the fitting tool of 

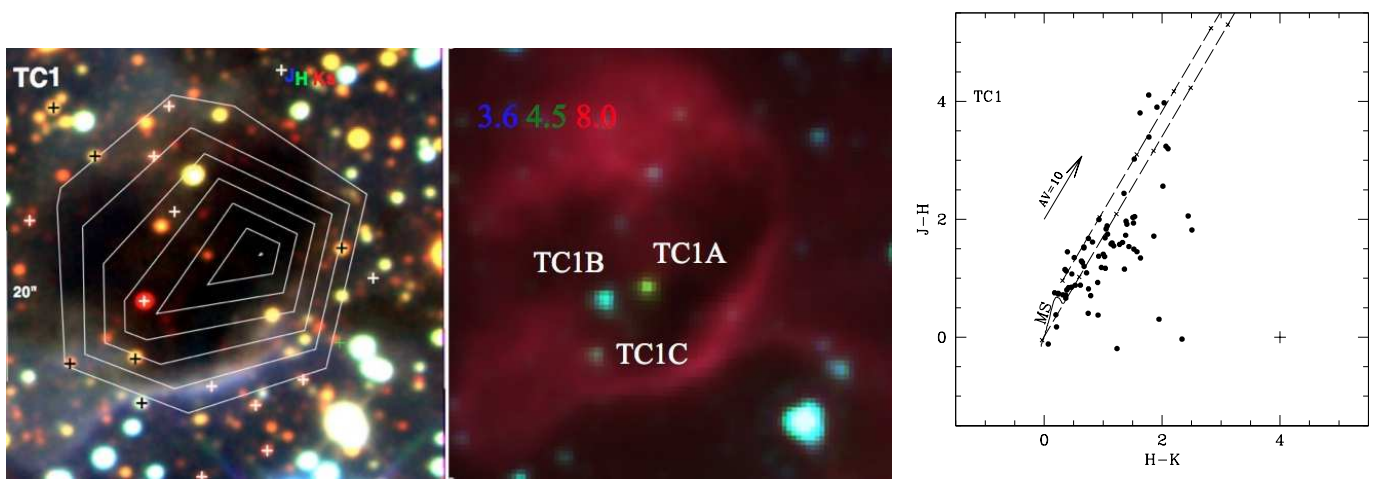

Figure 3: Left panel:JHK (blue, green, red) and IRAC/Spitzer true color images of a region of size $1^{\prime} \times 1^{\prime}$ around TC1. North is at the top and east to the left. The contours are the $250 \mu \mathrm{m}$ Herschel emission. Right panel: $J-H$ versus $H-K_{s}$ diagram of the near-IR sources observed in TC1.

[13]. The derived parameters are reported in Table 2.
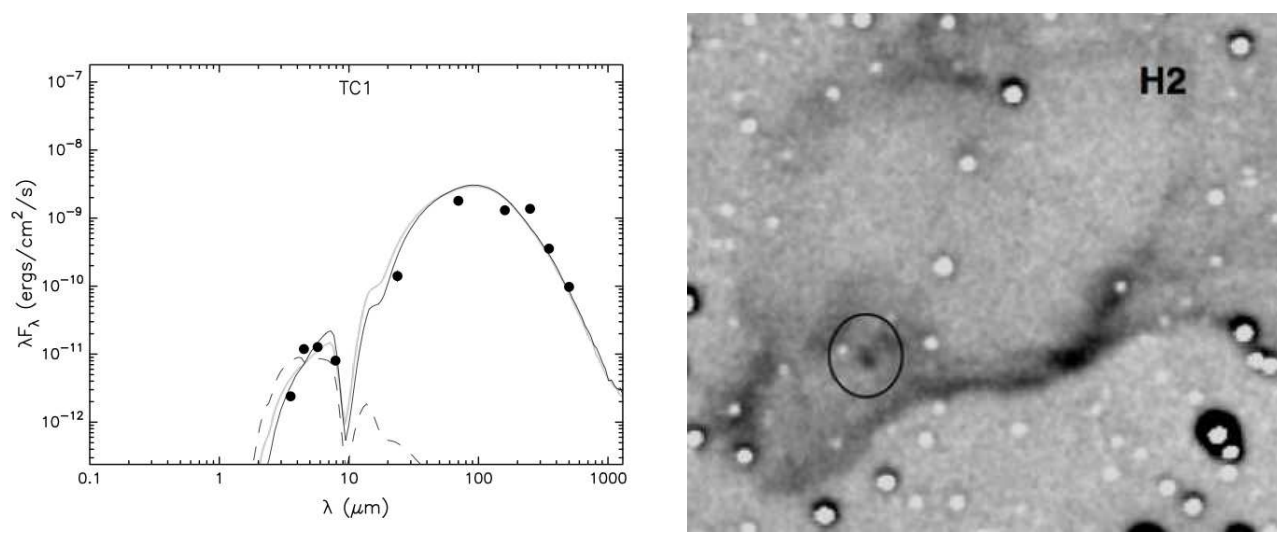

Figure 4: Left panel: Spectral energy distribution (SED) of TC1A. The best-fit models of the SED derived from fitting tool [13] is over-plotted as solid line. Right panel: $\mathrm{H}_{2}$ image of TC1.

The $\mathrm{H}_{2}$ image illustrated in Fig.4 (Right panel) shows a filamentary structure and an $\mathrm{H}_{2}$ knots (ellipse in Fig.4) probably excited by the young star TC1C that is the counterpart of the X-ray source detected by Chandra .

\subsection{The Trifid condensation TC2}

We have found just a small number of sources with near-IR excess toward the dense core TC2 as shows the $J-H$ versus $H-K_{s}$ diagram (Figure 5 Right panel ). In addition we have identified the very red source TC2A with a strong near-IR excess and with the IRAC counterpart as the millimeter source (Figure 5 Left panel). . As in the previous dense condensation, we have obtained the SED including near-IR, Spitzer, Herschel, and millimeter data (Figure 6 Left panel ). From the best fit of the SED with the model [13] we have derived the phisycal parameters of TC2A reported in Table 2.

An $\mathrm{H}_{2}$ knots is present in our $\mathrm{H}_{2}$ image shown in Figure 6 (Right panel). The protostar TC2A is responsible for the observed knots. 
Table 2: Physical parameters of source TC1A and source TC2A derived from model [13].

\begin{tabular}{lll}
\hline Parameters & TC1A & TC2A \\
\hline Stellar Mass $\left(\mathrm{M}_{\text {sun }}\right)$ & 4.7 & 3.1 \\
Stellar Temperature $(\mathrm{K})$ & 12000 & 4228 \\
Envelope Accretion Rate $\left(\mathrm{M}_{\text {sun }} / \mathrm{yr}\right)$ & $2.310^{-3}$ & $8.2210^{-4}$ \\
Envelope Cavity Angle $(\mathrm{deg})$ & 14 & 6.8 \\
Disk Mass $\left(\mathrm{M}_{\text {sun }}\right)$ & 0.11 & $1.0810^{-3}$ \\
$A_{V}$ & 59 & 23 \\
$L_{\text {bol }}\left(\mathrm{L}_{\text {sun }}\right)$ & 308 & 139 \\
\hline
\end{tabular}
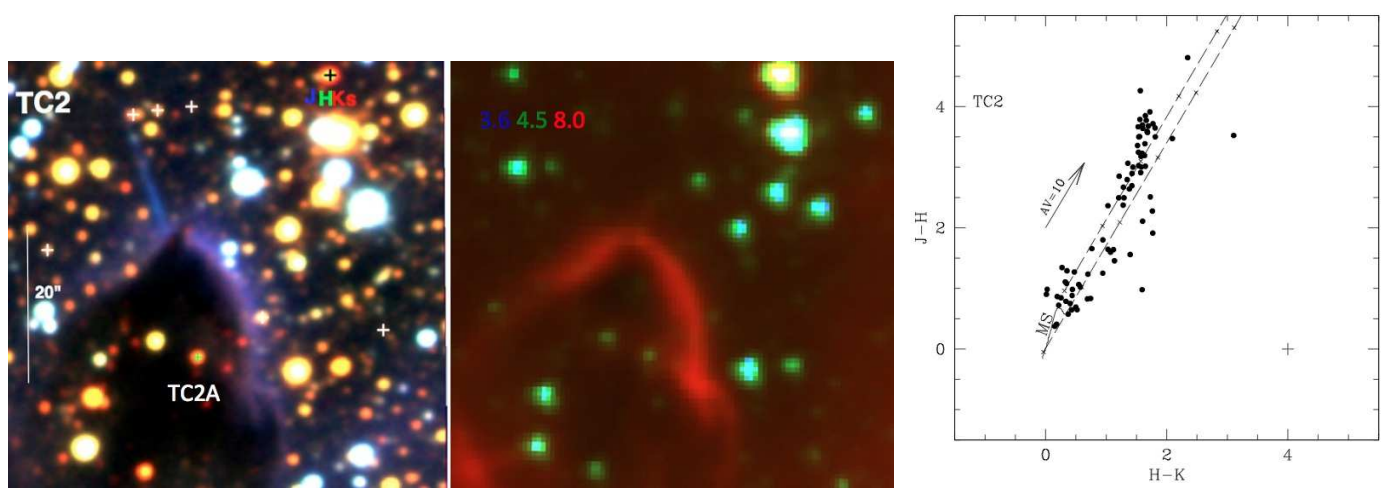

Figure 5: Left panel: $J H K_{S}$ (blue, green, red) and IRAC/Spitzer true color images of a region of size 1' $\times$ 1' around TC2. North is at the top and east to the left. Right panel: $J-H$ versus $H-K_{s}$ diagram of the near-IR sources observed in TC2.
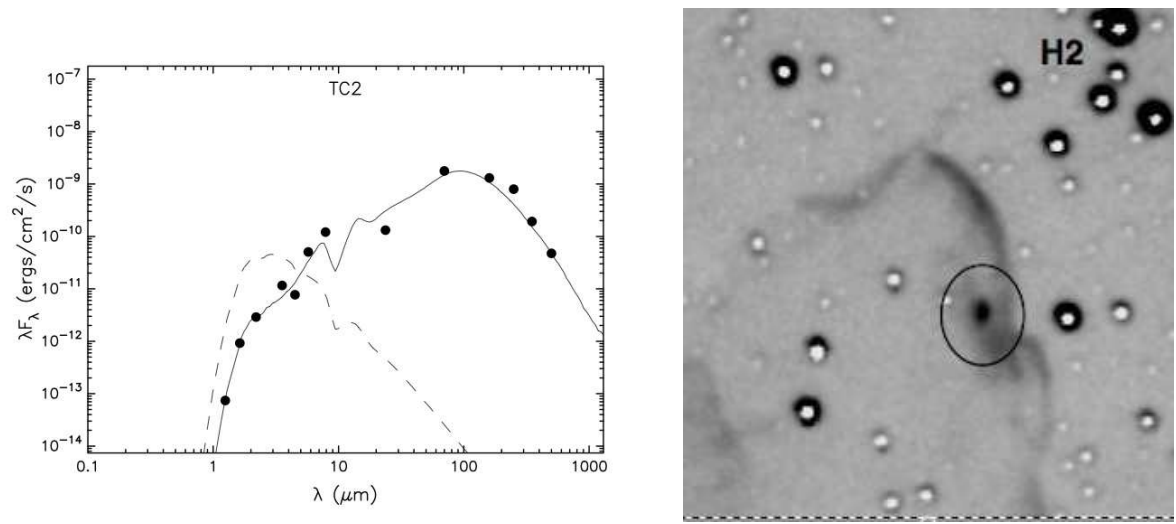

Figure 6: Left panel:Spectral energy distribution (SED) of TC2A. The best-fit models of the SED derived from fitting tool [13] is over-plotted as solid line. Right panel: $\mathrm{H}_{2}$ image of TC2. 


\subsection{The Trifid condensation TC3}

No evidence of a young stellar cluster is associated with TC3 as shown in Figure 7. A source detected in the mid- IR and at $\mathrm{mm}$ is found very close to the center of the dense core. This is a Class 0 protostellar object with $\mathrm{A}_{V}=50$ and $\mathrm{L}_{\text {tot }}=182$ Lsun. Its SED is illustrated in Fig 8 (Left panel).
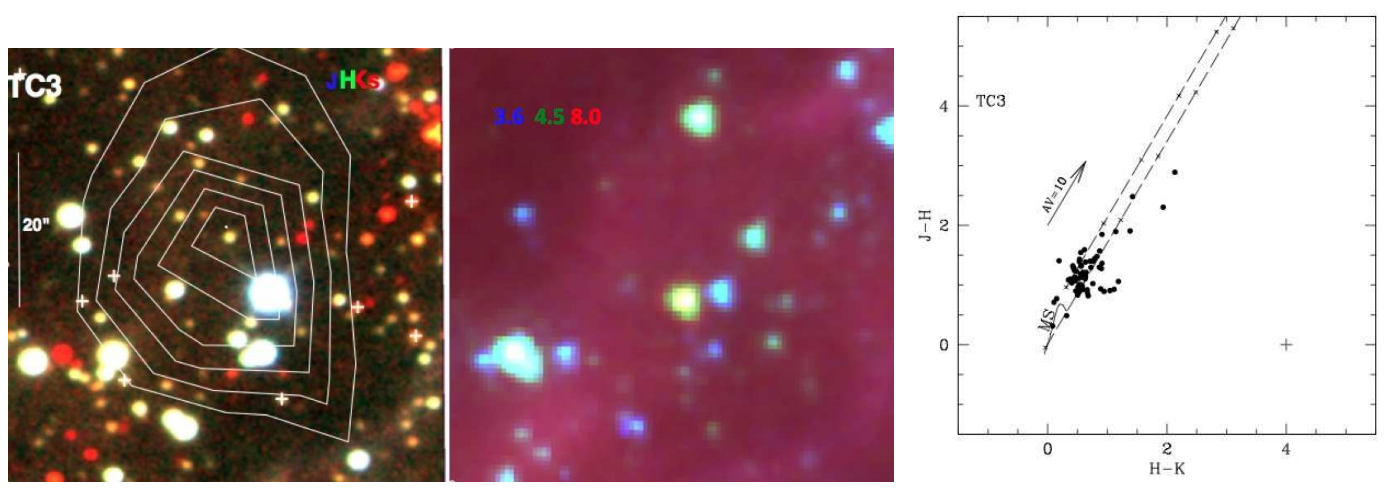

Figure 7: Left panel: $J H K_{s}$ (blue, green, red) and IRAC/Spitzer true color images of a region of size 1' $\times$ 1' around TC3. North is at the top and east to the left. Right panel: $J-H$ versus $H-K_{s}$ diagram of the near-IR sources observed in TC3.

At difference of the dense cores TC1 and TC2, the $\mathrm{H}_{2}$ image of TC3 (Fig.8 Right panel) does not show the presence of knots but just a filament in the south-west of the core.
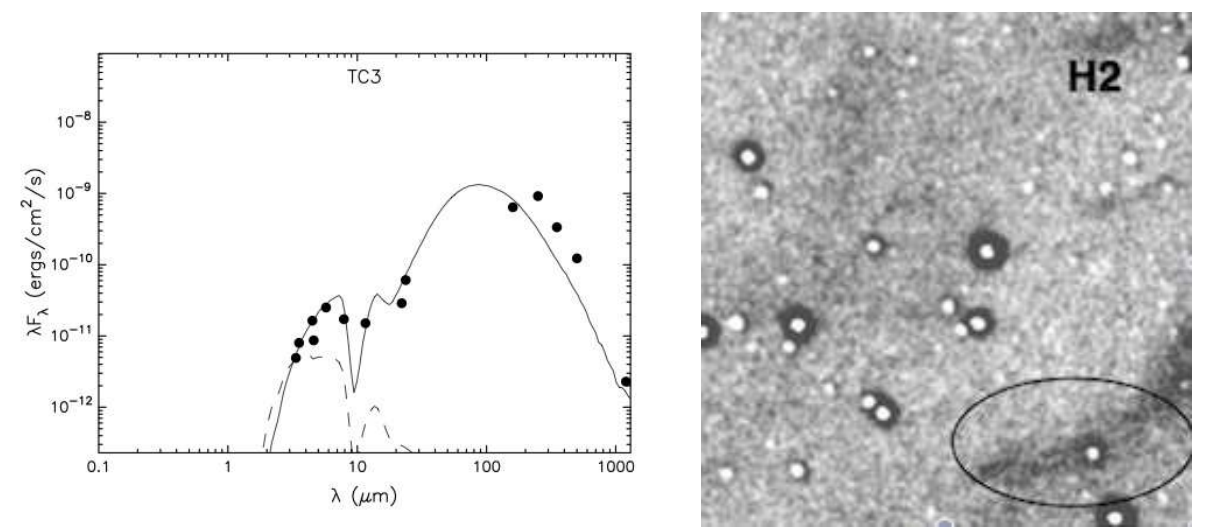

Figure 8: Left panel:Spectral energy distribution (SED) of TC3. The best-fit models of the SED derived from fitting tool [13] is over-plotted as solid line. Right panel: $\mathrm{H}_{2}$ image of TC3.

\subsection{The Trifid condensation TC4}

Herschel images of this condensation, show a very complex structure (see Fig.1) with at least three sub-condensations. We have analyzed a field of 2' $\times 2$ ' containing the three condensations. Figure 9 (Left panel) shows the JHK color coded image compared with the IRAC/Spitzer color coded image. A cluster of young star is present in TC4 as shows the color color diagram of Fig.9 

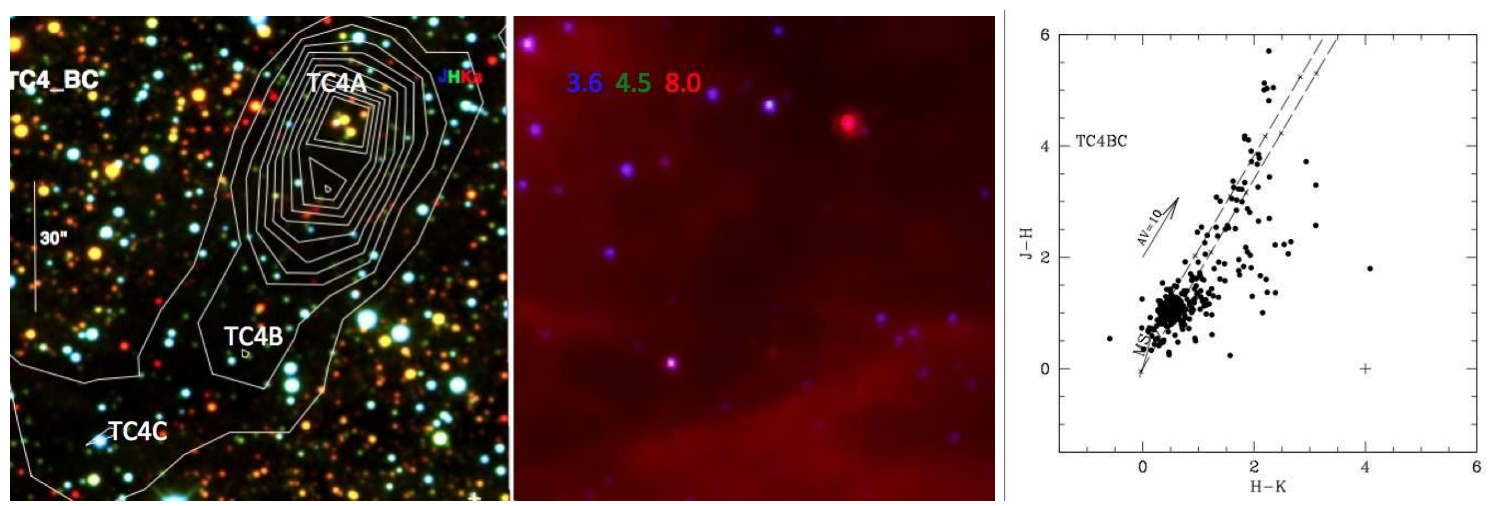

Figure 9: Left panel: $J H K_{S}$ (blue, green, red) and IRAC/Spitzer true color images of a region of size 2' $\times$ 2' around TC4. North is at the top and east to the left. Right panel: $J-H$ versus $H-K_{s}$ diagram of the near-IR sources observed in TC4.

(Right panel). Particular interesting is the presence of a source with near-IR excess coincident with the far IR peak and with a very red IRC/Spitzer source. The derived SED is illustrated in Figure 10 Left panel. This source is responsible for the observed bipolar $\mathrm{H}_{2}$ jets (see Fig.10 Right panel)
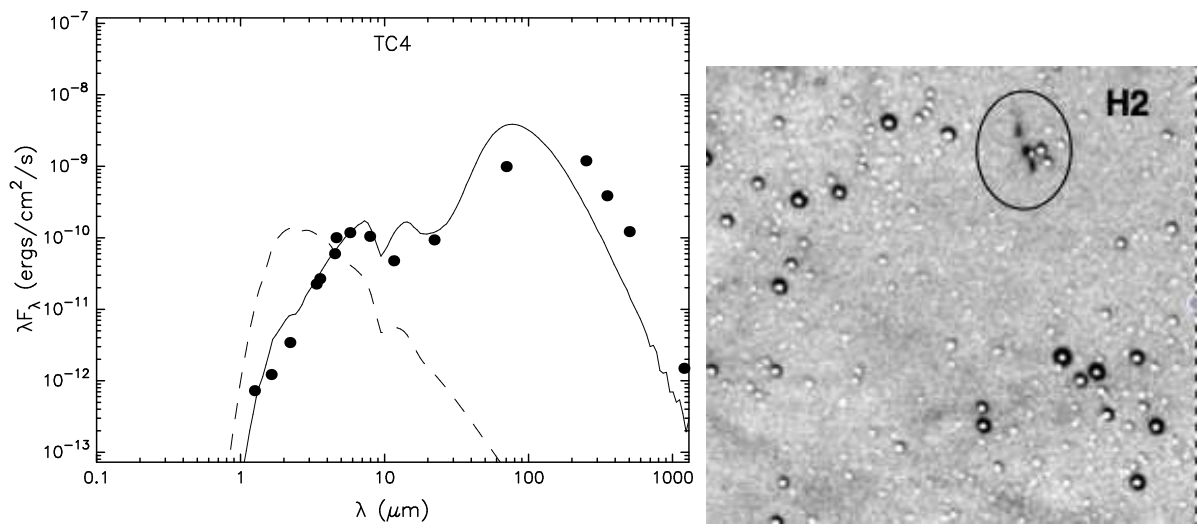

Figure 10: Left panel:Spectral energy distribution (SED) of TC4. The best-fit models of the SED derived from fitting tool [13] is over-plotted as solid line. Right panel: $\mathrm{H}_{2}$ image of TC4.

\section{Conclusions}

Our understanding of the young HII region M20 has made tremendous progress thanks to multi-wavelenghts observations .From a partial analysis of our observations of the Trifid Nebula we can derive the following conclusion:

1) Herschel images show the presence of 53 dense cores associated with the Trifid Nebula, mostly identified with the TC condensations detected at $\mathrm{mm}$ wavelength.

2) Different stages of star formation have been found associated to the dense condensations, from prestellar to protostellar cores with and witouth young stellar clusters.

3) In the dense cores TC1, TC2 and TC4 we have detected $\mathrm{H} 2$ jets excitated by protostellar objects.

4) M20 is a good example of massive-star forming region in a turbulent, filamentary molecular 
cloud. We propose that the nearby supernova remnant W28 could have triggered the formation of protostellar clusters in nearby dense cores of the Trifid.

\section{References}

[1] Cernicharo, J., et al. 1998, Science, 282, 462

[2] Lefloch, B., \& Cernicharo, J. 2000, ApJ, 545, 340

[3] Hester, J. J., et al. 1999, BAAS, 194, 681

[4] Rho, J., Reach, W., Lefloch, B., \& Fazio, G. 2006, ApJ, 643, 965

[5] Lefloch, B., Cernicharo, J., \& Pardo,J.R., 2008, A\&A, 489, 157

[6] Rho, J., Ram?õrez, S. V., Corcoran, M. F., Hamaguchi, K., \& Lefloch, B. 2004, ApJ, 607, 904

[7] Rho, J., Lefloch, B., Reach, W. T., \& Cernicharo, J. 2008, in Handbook of Star Forming Regions, vol II, ASP Conf. Ser., 5, 509

[8] Molinari S. et al., 2010, $A \& A, 518$, L100

[9] Traficante, A., Calzoletti, L., Veneziani, M., et al. 2011, MNRAS, 4162932

[10] Molinari, S., Faustini, F., Schisano, E., et al. 2011, A\&A, 530 A133

[11] Kovacs, Z., Mall,V., Bizenberger, P. et al. 2004, Proceedings of the SPIE, vol.5499, p.432

[12] Robitaille, T. P., Whitney, B. A., Indebetouw, R., et al. 2006, ApJS, 167256

[13] Robitaille, T. P., Whitney, B. A., Indebetouw, R., Wood, K. 2007, ApJS, 169328 